\title{
Complementos para Informática Industrial del perfil de Ingeniería de Computadores del Grado de Informática de la UGR: Controladores Lógicos Programables
}

\author{
M. Damas, O. Baños, G. Olivares, F. Gómez \\ Departamento de Arquitectura y Tecnología de Computadores. ETSI Informática y de \\ Telecomunicación. Universidad de Granada. \\ \{mdamas, oresti, gonzalo, frgomez\}@ugr.es
}

\begin{abstract}
Resumen. En este trabajo se presentan tanto la motivación como la metodología docente de la asignatura Controladores Lógicos Programables, una optativa de la materia de complementos para Informática Industrial del perfil de Ingeniería de Computadores del Grado de Informática de la ETSIIT de Granada, presentada en el marco de las III Jornadas de Coordinación Docente y de Empresas organizadas por el Departamento de Arquitectura y Tecnología de Computadores. Concretamente, en este trabajo se muestra el porqué de esta materia y asignatura, cómo se ha organizado para lograr los objetivos propuestos en la guía docente, la metodología y herramientas que se van a utilizar en la parte práctica de la asignatura, y finalmente las principales conclusiones de este trabajo.
\end{abstract}

Palabras Clave: Informática Industrial, Controladores Lógicos Programables, motivación del alumnado, laboratorio virtual, Ingeniería de Computadores.

\begin{abstract}
This paper shows the motivation and teacher methodology of the Programmable Logic Controller course, an elective of the Industrial Computer Complement subject of the Computer Engineering profile in Computer Sciences Grade of Granada ETSIIT, presented at the III Conference on Educational Coordination and Enterprises, organized by the Department of Computer Architecture and Computer Technology. Particularly here we show why this subject and course, how it is organized to achieve the objectives in the teaching guide, the methodology and tools to be used in the practical part of the course, and finally the main conclusions of this work.
\end{abstract}

Keywords: Industrial Computing, Programmable Logic Controllers, student motivation, virtual laboratory, Computer Engineering. 


\section{Introducción}

La puesta en marcha de nuevos Planes de Estudios en los que el Departamento de Arquitectura y Tecnología de Computadores (ATC) de la Universidad de Granada imparte asignaturas (Grados en Telecomunicaciones, Informática, Físicas, Electrónica industrial y Químicas), y la existencia en alguno de estos planes (Grado en Ingeniería Informática) de perfiles como el de Ingeniería de Computadores (IC), con una participación mayoritaria del mencionado Departamento, ponen de manifiesto la necesidad de desarrollar tanto actividades de coordinación de la docencia de las nuevas asignaturas, como de difusión de las perspectivas laborales asociadas a las competencias de esos perfiles.

Una de estas actividades es la organización anual de las Jornadas de Coordinación Docente y Empresarial del Departamento de Arquitectura y Tecnología de Computadores (JCDE) [1], y precisamente este trabajo se enmarca dentro de la III edición de estas jornadas, enfocadas en esta ocasión a coordinar y difundir las asignaturas optativas que imparte el Departamento de Arquitectura y Tecnología de Computadores en el Grado de Informática de la ETSIIT de la Universidad de Granada.

Concretamente, la optativa que se presenta aquí está incluida en la materia de Complementos de Informática Industrial del Perfil de Ingeniería de Computadores del Grado de Informática, junto con otra asignatura denominada Informática Industrial (ver figura 1). Ambas asignaturas tienes 6 créditos cada una y por tanto en total son 12 créditos los dedicados a complementos de Informática Industrial. Con este trabajo se pretende, en primer lugar, justificar la inclusión de esta materia como optativa en el Grado de Ingeniería Informática, ya que a priori parecería que las competencias que se puedan adquirir con ella pertenecen más al ámbito de la Ingeniería Industrial.

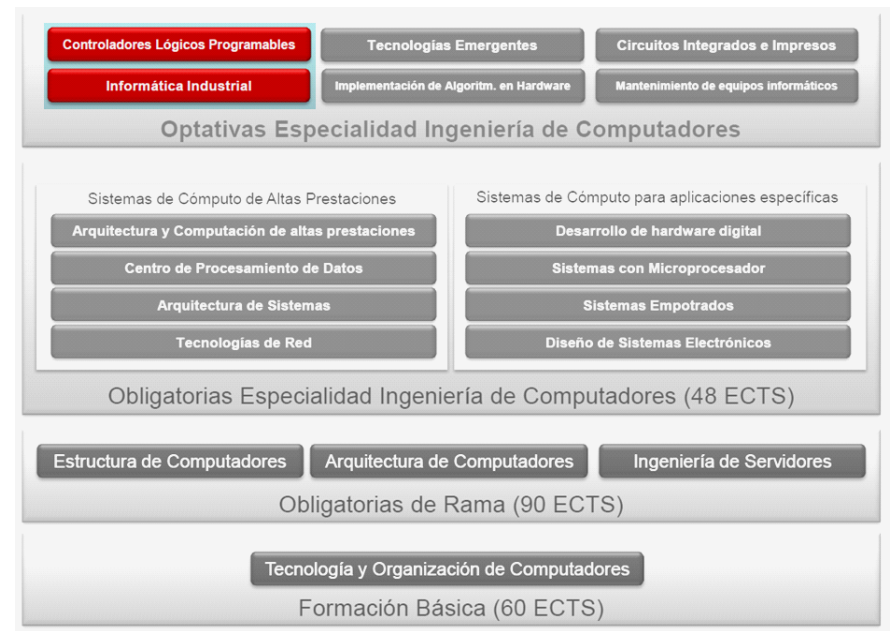

Figura 1. Complementos de Informática Industrial en el perfil de Ingeniería de Computadores en el Grado de Informática 
A continuación, y una vez argumentados los motivos por los que consideramos apropiado impartir dicha materia y en especial la asignatura Controladores Lógicos Programables en el Grado de Ingeniería Informática, también se muestra la metodología docente que se va a seguir y los equipos y herramientas que se van a utilizar en la parte práctica de dicha asignatura. Finalmente se presentan las conclusiones más importantes de este trabajo.

\section{2 ¿Por qué esta materia y asignatura?}

\section{1 ¿Por qué esta materia?}

En primer lugar, hay que decir que la informática y concretamente la ingeniería de computadores cada vez está más presente en el ámbito de la automatización industrial. Por ejemplo, las arquitecturas de los controladores que se utilizan en la industria cada vez tienen mayores prestaciones, he incluso nos encontramos con configuraciones paralelas y redundantes, por no hablar de que los paradigmas actuales, como por ejemplo el Cloud Computing, aunque más lentamente también están llegando al entorno industrial.

Además, las herramientas de programación y configuración de los equipos de automatización (PLC, DCS, PAC, etc.) [2], así como algunas aplicaciones específicas como los SCADA [3], el estándar de comunicaciones OPC [4] o las herramientas MES [5] que se utilizan actualmente en la industria, son cada vez más complejas y con mayores funcionalidades.

Por lo tanto, es de suponer que un ingeniero informático estará más familiarizado a herramientas y aplicaciones similares a estas, y seguro que les sacará un mayor rendimiento. En la figura 2 se muestran ejemplos de estas configuraciones y herramientas en el ámbito industrial que cada vez son más sofisticadas.

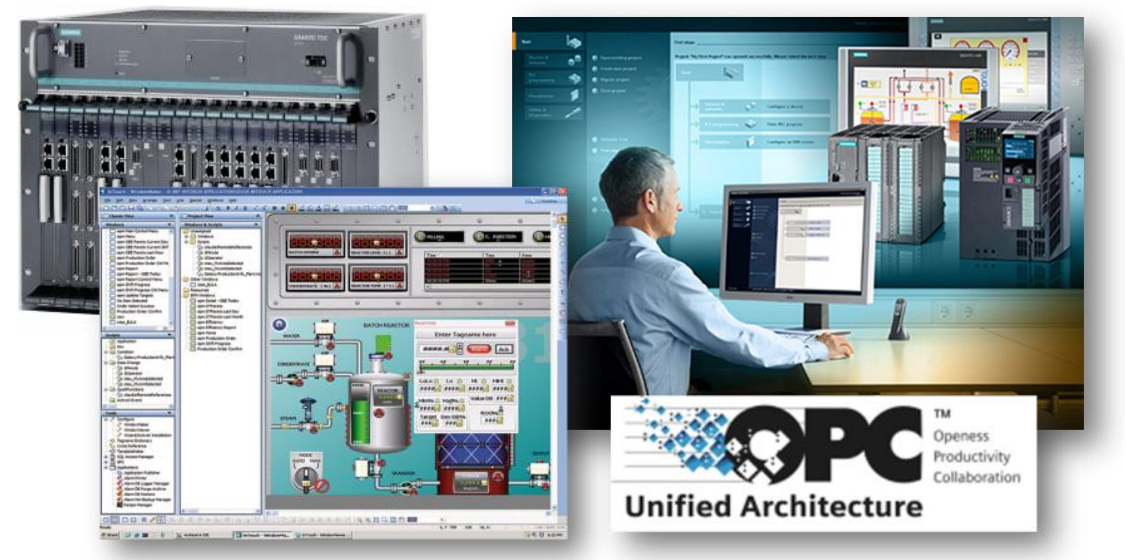

Figura 2. Ejemplos de equipos y herramientas actuales en la automatización. 


\section{2 ¿Por qué esta asignatura?}

Una vez introducida brevemente la motivación para cursar esta materia en el Grado de Informática, a continuación nos vamos a centrar en dar algunos motivos más por los que la asignatura Controladores Lógicos Programables se debe impartir dentro de esta materia:

- En primer lugar señalar que el concepto de PLC está muy extendido y su modelo en cuanto a diseño, programación y configuración es seguido por otros equipos similares: DCS, PAC, RTU, Slot-PLC, Soft-PLC, etc.

- Los PLC son los sistemas más utilizados en la automatización de proceso industriales, como lo demuestra la gráfica siguiente (figura 3) donde se puede observar el \% de estos sistemas instalados.

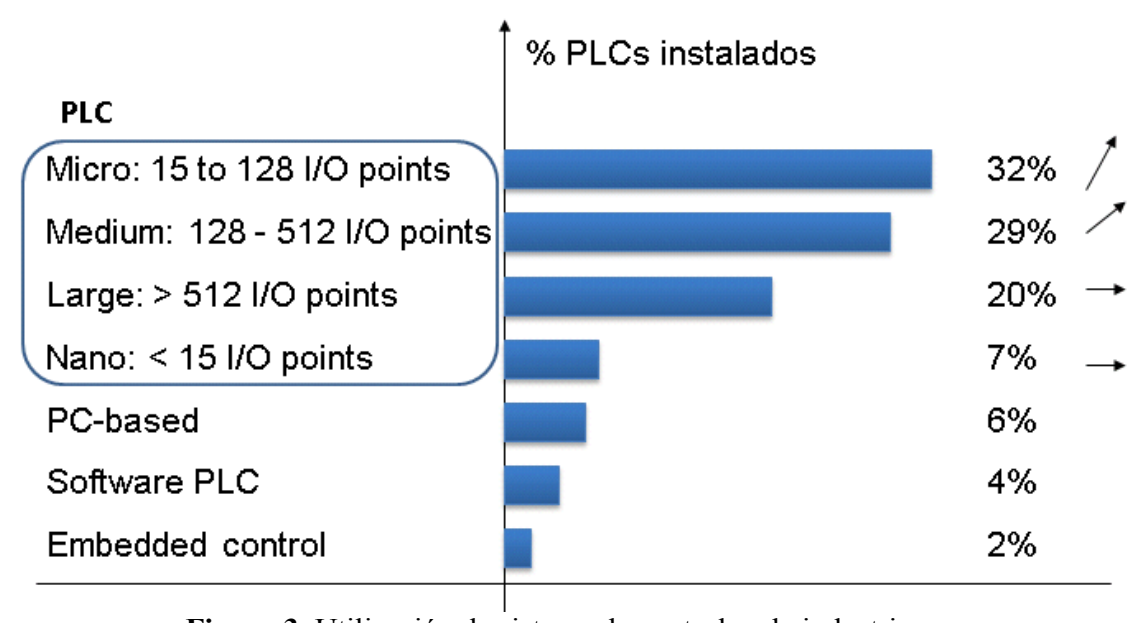

Figura 3. Utilización de sistema de control en la industria

(Fuente: Control Engineering, Reed Research, 2009).

- Los PLC además se utilizan en todo tipo de industrias: automatización de fábricas, procesos y edificios, industrias de generación y transporte de energía, etc.

- Los Ingenieros Informático con estos conocimientos complementan muy bien a los Ingenieros Industriales, como se ha podido constatar en empresas cercanas y pertenecientes al sector de la automatización industrial como ICR S.A., Akron, Ingenion Soluciones, etc.

- Otro motivo por el que impartir estas asignaturas es el de potenciar el área de Ingeniería de Sistemas y Automática del Departamento de Arquitectura y Tecnología de Computadores de la Universidad de Granada.

- Y finalmente indicar también que una asignatura optativa similar en la ingeniería informática a extinguir ha tenido hasta ahora una buena aceptación por parte de los alumnos de esta titulación, tal como se puede ver en la siguiente gráfica (figura 4), donde se puede apreciar que en término medio se han matriculado en torno a 35 alumnos. 


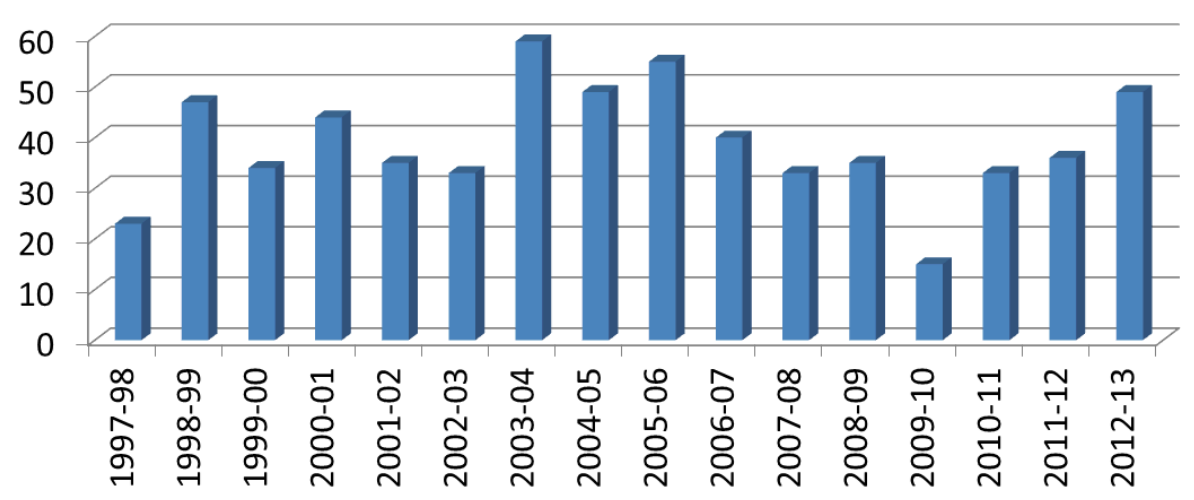

Figura 4. Número de alumnos matriculados en la asignatura PLC de la Ingeniería Informática a extinguir

\section{Temario Teórico y Práctico: Guía Docente}

En la guía docente elaborada para la asignatura Controladores Lógicos Programables aparecen los siguientes objetivos expresados como resultados de aprendizaje:

- Definir los conceptos básicos sobre automatismos en la industria.

- Conocer las arquitecturas típicas que se utilizan en el control de procesos industriales.

- Distinguir las características que diferencian a un PLC del resto de arquitecturas de control.

- Identificar las distintas partes que conforman la arquitectura interna de un PLC.

- Comprender el ciclo de funcionamiento interno de un PLC y su vinculación con el control en tiempo real.

- Diseñar un PLC teniendo en cuentas sus posibles configuraciones e interfaces de Entrada/Salidas y Específicas.

- Programar un PLC con los diferentes lenguajes que propone el estándar IEC 61131-3.

- Utilizar diferentes entornos de programación de PLC.

- Identificar el papel de los PLC en las redes de comunicaciones industriales.

- Conocer las redes comerciales más usadas en la automatización de procesos industriales.

En base a estos objetivos se ha planteado el siguiente temario teórico y práctico para esta asignatura, teniendo en cuenta que los temas teóricos y los seminarios necesarios para poder impartir las prácticas estén correctamente temporizados, es decir, que se impartan antes o como mucho al mismo tiempo. 


\section{TEMARIO TEÓRICO:}

Tema 1. Introducción al Control Industrial

1.1. Sistemas de Control

1.2. Automatismos cableados y programables

1.3. Arquitecturas de control: Unidades terminales remotas (RTU), Controladores lógicos programables (PLC), Controladores industriales, Ordenadores industriales (IPC), Controladores de Automatización Programables (PAC), Control basado en PC (SlotPLC, Soft-PLC), etc.

1.4. Centros de control y software SCADA

1.5. Fabricación integrada por computador (CIM)

Tema 2. Diseño de Automatismos

2.1. Automatismos combinacionales y secuenciales

2.2. Grafo de control etapa-transición (GRAFCET)

2.3. Puestas en marcha y paradas

2.4. Diseño de automatismos de procesos continuos

Tema 3. Controlador Lógico Programable

3.1. Arquitectura interna del PLC

3.2. Ciclo de funcionamiento y control en tiempo real

3.3. Configuración del PLC

3.4. Interfaces de Entrada/Salida y Específicas

Tema 4. Programación del PLC

4.1. Identificación de variables y asignación de direcciones

4.2. Lenguajes de programación

4.3. Programación de bloques funcionales

4.4. Entornos de programación

4.5. El estándar IEC 61131-3

Tema 5. Comunicaciones en los PLC

5.1. Enlaces estándar en el nivel físico

5.2. Redes de comunicación industriales

5.3. Ejemplos de Redes comerciales: Modbus, Profibus, ASI, CAN, HART, EtherCAT, etc.

Tema 6. Ejemplos de Aplicaciones de Automatización

6.1. Telecontrol del Ciclo Integral de Agua (Ciudad de Granada)

6.2. Control y Supervisión de una Estación de Esquí (Sierra Nevada)

6.3. Automatización de una fábrica de Papel (Torraspapel)

\section{TEMARIO PRÁCTICO:}

Seminario práctico 1: Utilización del editor de programa (STEP7) de los PLC del laboratorio Seminario práctico 2: Programación y simulación de los PLC del laboratorio (Siemens) Seminario práctico 3: Uso de la herramienta gráfica para diseñar un GRAFCET

Prácticas 1: Implementación guiada de una práctica para el control de una maqueta pequeña (maqueta elevador)

Prácticas 2: Programación de un automatismo sencillo (maqueta parking coches) con cualquiera de los lenguajes convencionales del estándar IEC-61131

Prácticas 3: Programación en GRAFCET de un proceso secuencial (maqueta almacenamiento y distribución de bloques)

Prácticas 4: Interconexión entre un PLC y un SCADA mediante el estándar OP 
Como se puede extraer de los seminarios y prácticas planteadas la idea es que el alumno pueda realizar el ciclo completo de desarrollo de sistemas de control industriales, representado gráficamente en la figura 5, tal como se hace en la industria en general. Es decir, que disponga de un proceso que automatizar conectado con un PLC real que se pueda programar con una herramienta profesional lo más completa posible, y que a su vez el PLC esté comunicado mediante el estándar OPC con un SCADA comercial para poder supervisar y controlar el proceso real. En este escenario, el software se puede utilizar sin problemas en las prácticas (ya que hay versiones demos y licencias para estudiantes de dichas herramientas), pero será difícil tener un PLC para cada alumno (por el elevado coste que esto supondría) y no digamos un proceso real. Por ello, y para que el alumno pueda realizar unas prácticas siguiendo este esquema, hemos ideado una metodología e implementado unas herramientas (que se describen en la siguiente sección) que nos van a ayudar a conseguirlo.

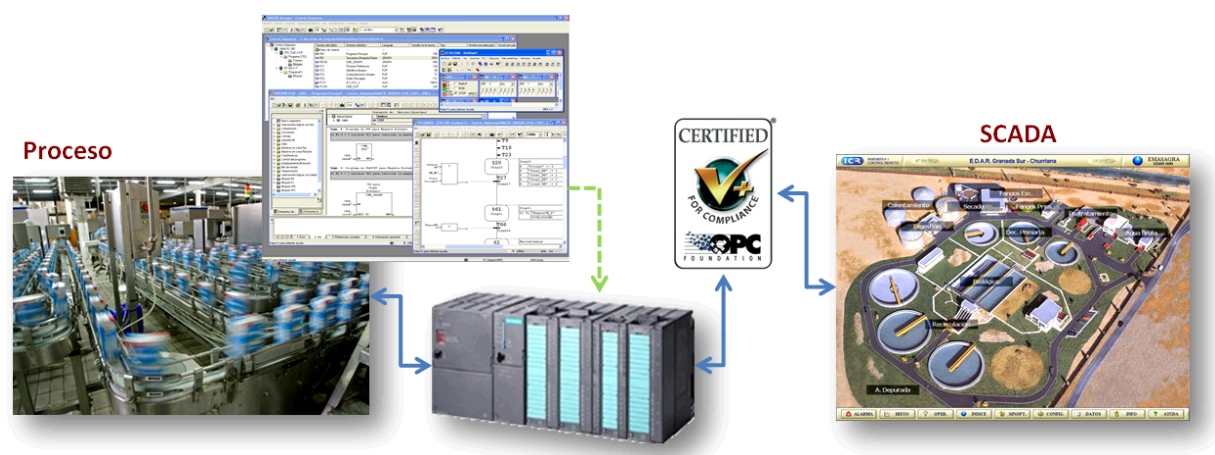

Figura 5. Ciclo completo de desarrollo de sistemas de control industriales

\section{Equipos, herramientas y metodología a utilizar}

Antes de describir los equipos, herramientas y metodología que se propone para esta asignatura, indicar que todo ello se ha adquirido y desarrollado bajo el paraguas de varios Proyectos de Innovación Docentes de la Universidad de Granada, coordinados por el Profesor Miguel Damas, y titulados:

1. Plataforma Docente Web para el Control de Maquetas de Simulación de Procesos Industriales (2007), galardonado con una mención honorífica en la convocatoria de Premios a la Innovación Docente 2008.

2. Laboratorio virtual para el ciclo completo de desarrollo de sistemas de control industriales (2011).

3. Nuevas metodologías de aprendizaje aplicadas a los sistemas de robótica industrial (2012).

Además, estas herramientas y metodología también han sido en parte publicadas en diversos congresos de docencia, tanto nacionales como internacionales $[6,7,8,9]$. 


\subsection{Equipos y Herramientas desarrolladas}

Utilizar un material adecuado de prácticas que motive al alumno en su aprendizaje es un factor muy importante a tener en cuenta. Por ejemplo, en [10] utilizan robots de LEGO para el estudio de sistemas embebidos o en [11] utilizan una maqueta de tres depósitos para asimilar de forma práctica conceptos fundamentales en el control de procesos. En nuestro caso, hemos optado por maquetas que emulan procesos industriales reales, ya que consideramos que son idóneas para la formación de técnicos en control de procesos. Concretamente, se han elegido varias maquetas fabricadas por Staudinger (ver figura 6), disponibles actualmente en el laboratorio de Control de la ETS de Ingeniería Informática y de Telecomunicación de la Universidad de Granada (ETSIIT).
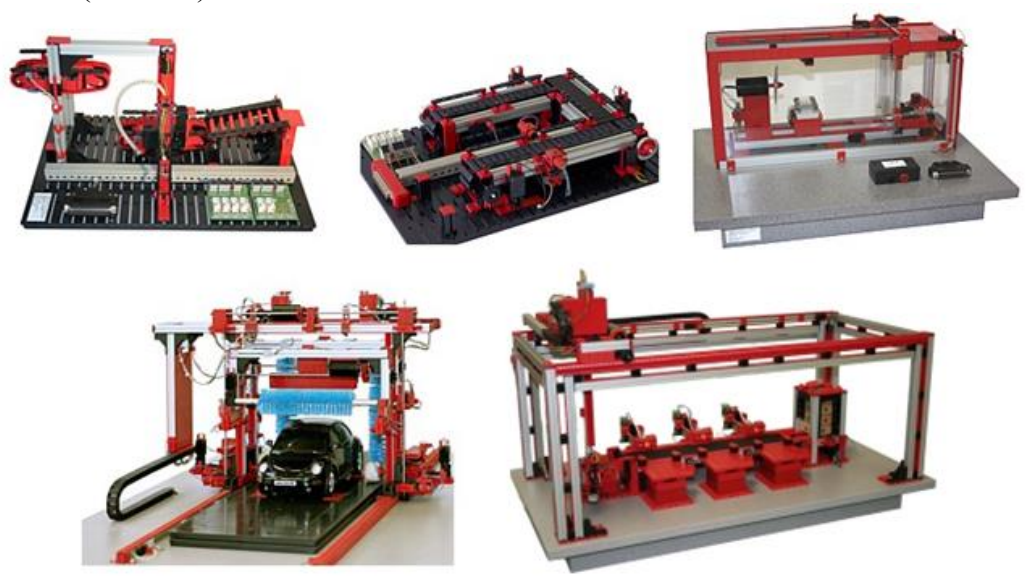

Figura 6. Algunas de las maquetas disponibles en el laboratorio

El laboratorio dispone también de un PLC de Siemens (muy utilizado en la industria en general), concretamente uno de la serie S7-300 (gama media). Se ha seleccionado este controlador porque se trata de unos de los fabricantes con mayor cota de mercado actualmente, y porque también proporciona un simulador de sus PLC (S7-PLCSim) y un ActiveX (S7-ProSim) que nos va a permitir integrar este simulador con otras aplicaciones: Maquetas virtuales y Servidor OPC desarrollados en nuestro caso.

\section{Maquetas virtuales}

Son programas que permiten emular gráficamente el comportamiento de las maquetas reales (ver figura 7). Es decir, que en función de las variables de salida que genera el programa realizado por el alumno y que se ejecuta en el simulador del controlador (S7-PLCSim), la maqueta virtual genera las variables de entrada necesarias sobre el simulador del controlador, para que este se comporte como si realmente estuviera conectado a la maqueta real. Además, la maqueta virtual muestra gráficamente los resultados de la ejecución de los programas para que el alumno compruebe si su práctica está funcionando correctamente o no. Algunas de las características y funciones más interesantes que ofrecen dichas maquetas virtuales son: 
- Representación gráfica y animada acorde con la maqueta real simulada.

- Fácil interconexión con el simulador del controlador.

- Parametrización de las variables de los sensores y actuadores de las maquetas.

- Configuración de distintas posiciones iniciales de partida para que los programas de control de los alumnos se comporten de forma adecuada.

- Detección automática de errores de funcionamiento, con notificación de mensajes explicativos para que el alumno pueda depurar sus prácticas. Es decir, un supervisor de acciones no permitidas genera mensajes de errores a los alumnos cuando intentan realizar una operación que pueda causar un daño a la maqueta real o que no esté permitida según el funcionamiento programado.

- Autoevaluación de las prácticas de los alumnos, con indicación de la puntuación final en función de las iteraciones y distintas situaciones iniciales realizadas con éxito.

- Almacenamiento y recuperación del estado actual de la ejecución: direccionamiento de variables, configuraciones iniciales definidas, parámetros de autoevaluación, incidencias y errores detectados, etc.

Destacar sobre estas maquetas virtuales la funcionalidad de autoevaluación de las prácticas de los alumnos, donde se puede configurar distintas situaciones de partida. Por ejemplo, para la maqueta elevador, se puede seleccionar que inicialmente el elevador o el contenedor puedan estar en cualquier posición, lo cual influirá en la nota final que se obtenga. También podemos indicar el número de iteraciones (ejecuciones del programa desde distintas posiciones de partida de la maqueta) que se van a realizar y el tiempo de cada iteración. Al final de la autoevaluación se muestra una ventana en la que se indica la puntuación final, el número de iteraciones erróneas, el número de iteraciones correctas y la ventana de incidencias para que podamos analizar el porqué de las iteraciones erróneas.
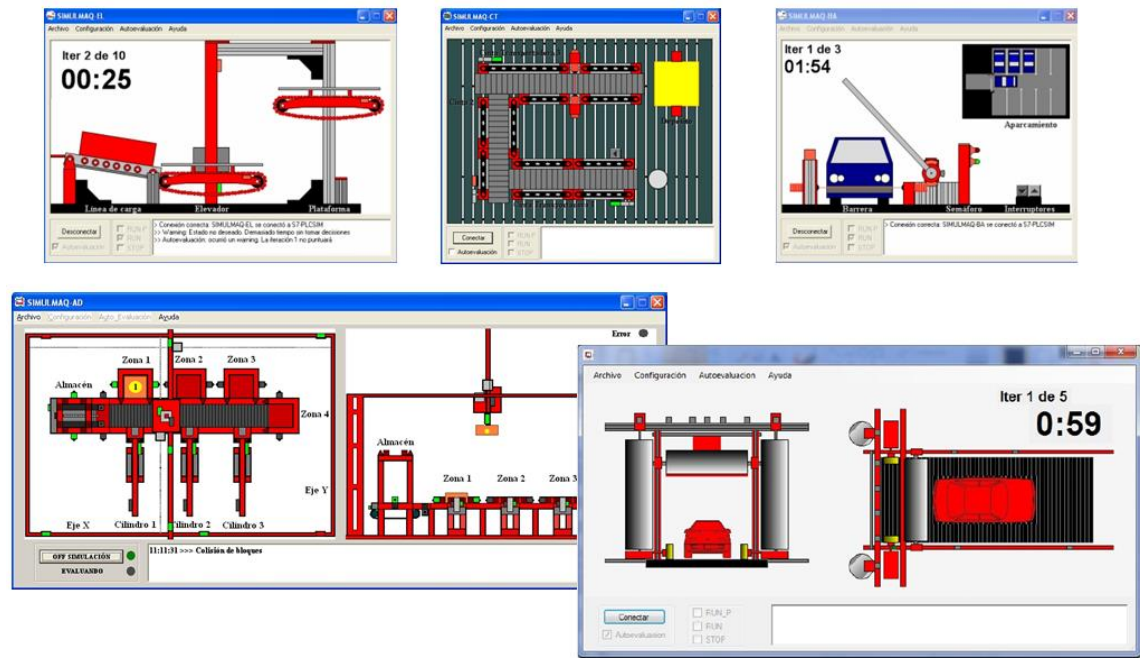

Figura 7. Maquetas virtuales desarrolladas para el laboratorio virtual: Maqueta elevador, maqueta cintas transportadoras, maqueta aparcamiento de coches, maqueta distribución y almacenamiento de bloques, y maqueta lava coches. 


\section{Servidor OPC-PLCSim}

OPC (OLE/COM for Process Control) es un mecanismo de comunicación que permite la interconexión y el intercambio de datos entre dispositivos y aplicaciones software, soportado por la OPC Foundation, y que se ha convertido en el estándar para resolver la interoperabilidad en el entorno industrial. Por tanto, se ha desarrollado un servidor de comunicaciones según este estándar OPC que nos permite comunicarnos por un lado con el simulador de controladores de Siemens (S7PLCSim) a través del ActiveX que proporciona el propio fabricante, y por otro lado con cualquier software de supervisión y control del mercado (SCADA) que implemente un cliente OPC.

\subsection{Metodología}

En cuanto a la metodología que se propone, como se ha indicado en la guía docente de la sección 3, se basa en una serie de seminarios y prácticas, acordes con el temario teórico y una serie de herramientas desarrolladas (descritas en la sección anterior), para que el alumno pueda abordar de forma práctica todas las fases necesarias a la hora de realizar un sistema completo de automatización industrial. Es decir, que el alumno pueda realizar prácticas no solo de la programación de los equipos de control sino también de la configuración del software de supervisión y control necesarios en las salas de control, así como también de la intercomunicación entre ambos, y siempre utilizando los equipos (PLCs), software (SCADA y Entornos de programación) y protocolos (OPC) que más se usan actualmente de forma comercial en la industria.

Las clases prácticas del curso comienzan con varios seminarios dedicados a introducir los entornos de programación y los lenguajes del estándar IEC 61131-3 [12] que se utilizan en la automatización industrial. Concretamente, se imparte un seminario sobre cómo se utiliza el editor de programa STEP7 de los PLC de Siemens, y otro sobre cómo se programan dichos PLC, y en especial de cómo se realiza un SFC (Sequential Function Chart).

Para completar los seminarios anteriores, a continuación en las prácticas 1 y 2 se realizan implementaciones guiadas por el profesor utilizando maquetas sencillas con el objetivo de ilustrar la utilización de elementos de programación habituales en los automatismos industriales (biestables, temporizadores y contadores), la ventaja de utilizar SFC, así como para explicar el manejo de las maquetas virtuales. Concretamente en esta fase se usan la maqueta elevador, la maqueta aparcamiento de coches, y la maqueta cintas transportadoras.

En la práctica 3 se le propone al alumno que implemente desde el principio un programa para el control de una maqueta más compleja, utilizando los lenguajes y herramientas ya introducidos en las fases anteriores. Para ello se utilizan las maquetas virtuales de distribución y almacenamiento de bloques y la de lava coches. Con dichas maquetas virtuales el alumno puede probar y depurar su programa cuantas veces quiera sin dañar las maquetas reales. Además, una vez tenga depurada su implementación, el alumno podrá lanzar una autoevaluación que le indicará automáticamente una puntuación final orientativa del grado de corrección del programa. Con esta práctica se pretende que el alumno afiance sus conocimientos de 
programación de automatismos utilizando un entorno de programación comercial, así como los lenguajes del estándar 61131-3, y en especial, el SFC para implementar las tareas secuenciales de los procesos de fabricación industriales.

Finalmente, en la última práctica se completa el ciclo de desarrollo en la automatización de un proceso industrial (ver figura 8) introduciendo el estándar de comunicaciones OPC, y el software para la supervisión y control de procesos (SCADA). Para ello se utiliza un servidor OPC, implementado a propósito para este laboratorio virtual, que permite comunicar el simulador del PLC con el SCADA. En esta práctica, el alumno debe desarrollar una aplicación de supervisión y control para cualquiera de las maquetas disponibles, utilizando una herramienta comercial (como por ejemplo InTouch de Wonderware) y un servidor OPC-PLCSim para acceder a las variables del PLC simulado, de la misma forma que se haría en una aplicación real.

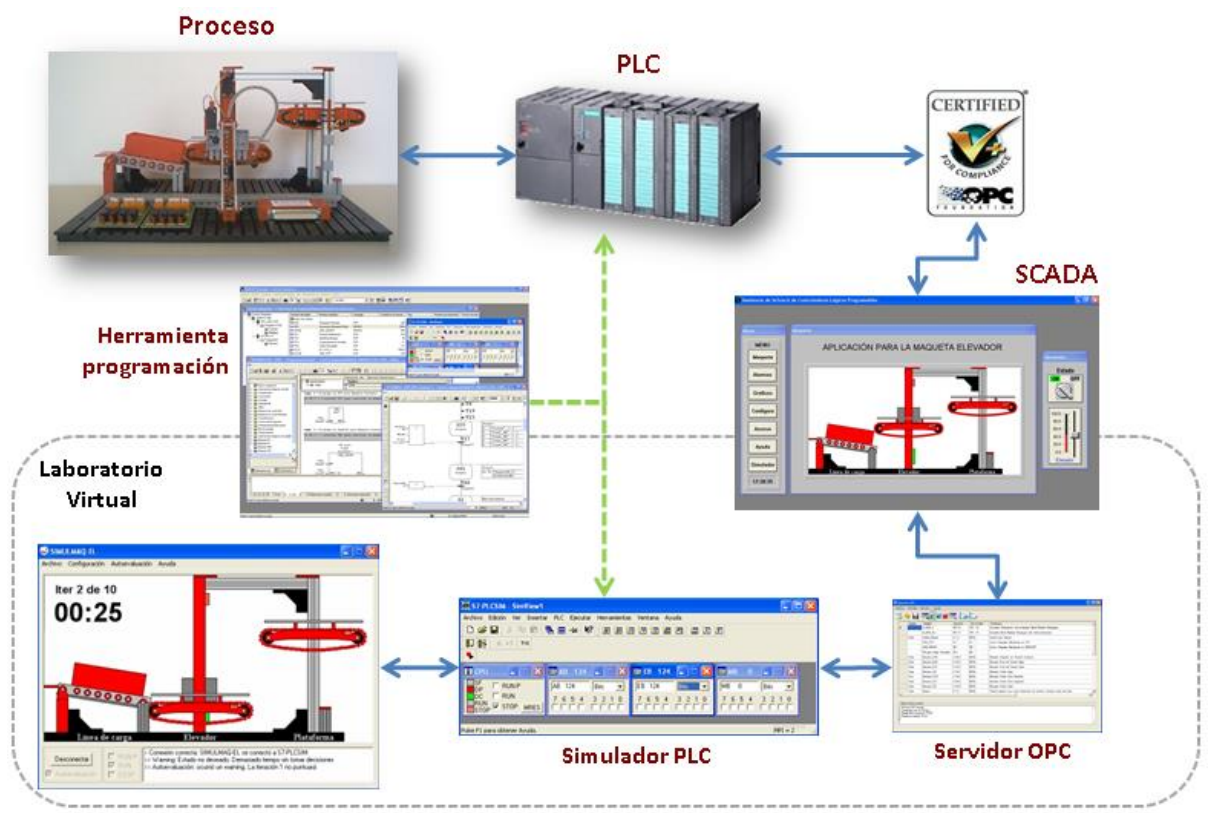

Figura 8. Esquema general de trabajo con el laboratorio virtual propuesto

\section{Conclusiones}

En este trabajo en primer lugar se ha presentado la asignatura Controladores Lógicos Programables como optativa del Grado de Ingeniería Informática de la Universidad de Granada, justificando su impartición en esta titulación dentro de la materia de complementos de Informática Industrial. A continuación, se han presentado tanto la metodología como los equipos y herramientas que se van a utilizar en dicha asignatura, en torno a un laboratorio virtual desarrollado para ello. 
El facilitar un laboratorio virtual donde se pueda trabajar como si se estuviera en un entorno industrial real es fundamental para poder evaluar adecuadamente que los alumnos hayan adquirido una competencia importante para ellos.

La metodología propuesta se ha presentado tanto desde el punto de vista técnico como educacional. Concretamente, se han mostrado los componentes tanto hardware como software que conforman el sistema, y se han descrito algunas de las maquetas con las que se trabajan. También, y de forma muy resumida, se ha presentado el escenario pedagógico que se sigue, donde se utilizan adecuadamente las herramientas presentadas en este trabajo.

La posibilidad de realizar prácticas en las distintas fases de realización de un proyecto (programación de controladores, interfaces de comunicaciones y software de supervisión y control) hace que el alumno tenga una visión completa del ciclo de desarrollo que todo ingeniero ha de realizar en la automatización de cualquier proceso industrial.

Las herramientas presentadas permiten que el alumno pueda organizarse mejor y eliminar barreras físicas (desplazamientos) a la hora de realizar las prácticas de asignaturas que hasta ahora necesitaban una presencia forzosa por la utilización de equipos específicos. También se minimiza el número de pruebas sobre las maquetas reales evitando daños y pérdidas de tiempo tanto del alumno como del profesor que ha de evaluar las prácticas.

Se debe de destacar también que la función de auto evaluación que incorpora la herramienta permite de forma automática realizar un chequeo exhaustivo de las prácticas proporcionando al final una calificación orientativa al alumno.

Luego, el esquema de trabajo presentado para la asignatura Controladores Lógicos Programables tiene, a nuestro parecer, un gran potencial didáctico, ya que permite que el alumno pueda trabajar con herramientas de programación y equipos reales que se emplean habitualmente en el control de cualquier proceso industrial, sin que su disponibilidad, coste, tamaño, o ubicación sean un problema.

Agradecimientos. Al Secretariado de Innovación Docente de la Universidad de Granada por los Proyectos de Innovación Docente concedidos que han posibilitado la adquisición de los equipos necesarios para desarrollar la metodología docente presentada en este trabajo.

\section{Referencias}

1. M. Damas, J. Ortega, O. Baños: "Una iniciativa para la coordinación y difusión de la docencia del perfil de Ingeniería de Computadores del Grado de Informática de la UGR". Enseñanza y Aprendizaje de Ingeniería de Computadores. Revista de Experiencias Docentes en Ingeniería de Computadores. ISSN: 2173-8688, No2, pp.73-80, 2012.

2. J.A. Rehg, G.J. Sartori, "Programmable Logic Controllers", ISBN: 978-0135048818, Prentice Hall, 2 edition, 2008.

3. S.A. Boyer, "SCADA: Supervisory Control and Data Acquisition", ISBN: 9781556178771, ISA. 3 edition, 2004. 
4. W. Mahnke, S. Leitner, M. Damm, “OPC Unified Architecture”, ISBN: 978-3540688983, Springer, 1 edition, 2009.

5. H.Meyer: "Manufacturing Execution Systems: Optimal Design, Planning, and Deployment". ISBN: 9780071623834, McGraw-Hill Companies, Inc., 2009.

6. M.Damas, H.Pomares, I.Rojas: "A new E-learning Strategy for Industrial Computation Students". IASK International Conference Teaching and Learning, Aveiro (Portugal), 2008.

7. M.Damas, H.Pomares, I.Rojas: "Use of an E-Learning Platform to Create a Virtual Lab in Computer and Electrical Engineering Courses". Improving University Teaching IUT'08, Transforming Higher Education Teaching and Learning in the 21st Century, Glasgow (United Kingdom), 2008.

8. M.Damas, G.Olivares, H.Pomares, A.Olivares: "Laboratorio Virtual basado en Web para el ciclo completo de desarrollo de sistemas de control industriales". Workshop en Informática Industrial (WIIND), ISBN: 84-692-2381-9, Universidad Rey Juan Carlos, Madrid, 2009.

9. M.Damas, H.Pomares, G.Olivares: "Innovación en la docencia práctica de asignaturas relacionadas con la informática industrial". $1^{\text {a }}$ Jornadas Andaluzas de Innovación Docente Universitaria. ISBN: 978-84-692-7263-3, Córdoba, 2009.

10. S. H. Kim, J. W. Jeon, "Introduction for Freshmen to Embedded Systems Using LEGO Mindstorms", IEEE Transactions on Education, Vol. 52, no. 1, pp. 99-108, 2009.

11. R. Dormido, H. Vargas, N. Duro, J. Sánchez, S. Dormido-Canto, G. Farias, F. Esquembre, and S. Dormido, "Development of a Web-Based Control Laboratory for Automation Technicians: The Three-Tank System", IEEE Transactions on Education, Vol. 51, no. 1, pp.35-44, 2008.

12. J. Karl-Heinz, M. Tiegelkamp, "IEC 61131-3: Programming Industrial Automation Systems", ISBN: 978-3540677529, Springer, 1 edition, 2001.

13. C. Buiu, "Hybrid Educational Strategy for a Laboratory Course on Cognitive Robotics", IEEE Transactions on Education, Vol. 51, no. 1, pp.100-107, 2008.

14. M. Duarte, B. P. Butz, S. M. Miller, A. Mahalingam, "An Intelligent Universal Virtual Laboratory (UVL)", IEEE Transactions on Education, Vol. 51, no. 1, pp. 2-9, 2008.

15. B. Barros, T. Read, M. F. Verdejo, "Virtual Collaborative Experimentation: An Approach Combining Remote and Local Labs", IEEE Transactions on Education, Vol. 51, no. 2, pp.242-250, 2008

16. Z. Nedic, J. Machotka, A. Nafalski, "Remote laboratories versus virtual and real laboratories," in Proc. 33rd Frontiers in Education Conf., Boulder, CO, pp. T3E.1-T3E.6, 2003. 